\title{
Effective simultaneous approximation of complex numbers by conjugate algebraic integers
}

\author{
by
}

\author{
G. J. Rieger (Hannover)
}

We study effectively the simultaneous approximation of $n-1$ different complex numbers by conjugate algebraic integers of degree $n$ over $\mathbb{Z}(\sqrt{-1})$. This is a refinement of a result of Motzkin [2] (see also [3], p. 50) who has no estimate for the remaining conjugate. If the $n-1$ different complex numbers lie symmetrically about the real axis, then $\mathbb{Z}(\sqrt{-1})$ can be replaced by $\mathbb{Z}$.

In Section 1 we prove an effective version of a Kronecker approximation theorem; we start with an idea of H. Bohr and E. Landau (see e.g. [4]); later we use an estimate of A. Baker for linear forms with logarithms. This and also Rouché's theorem are then applied in Section 2 to give the result; the required irreducibility is guaranteed by the Schönemann-Eisenstein criterion.

1. On the Kronecker approximation theorem. Let $k \in \mathbb{N}:=\{n \in$ $\mathbb{Z} \mid n>0\}, v \in \mathbb{N}, U \in \mathbb{R}, U \geq 1, i:=\sqrt{-1}, e(x):=\exp (2 \pi i x)(x \in \mathbb{R})$; let $p_{1}<p_{2}<\ldots<p_{k}$ be primes and

$$
\begin{gathered}
u_{\nu} \in \mathbb{Z}, \quad 0<\left|u_{\nu}\right| \leq U, \quad \beta_{\nu} \in \mathbb{R} \quad(\nu=1, \ldots, k), \\
f(t):=1+e(t)+\sum_{\nu=1}^{k} e\left(t \frac{u_{\nu}}{v} \log p_{\nu}-\beta_{\nu}\right) \quad(t \in \mathbb{R}) .
\end{gathered}
$$

With $\gamma_{-1}:=0, \beta_{-1}:=0, \gamma_{0}:=1, \beta_{0}:=0, \gamma_{\nu}:=\left(u_{\nu} / v\right) \log p_{\nu}(\nu=1, \ldots, k)$ we have

$$
f(t)=\sum_{\nu=-1}^{k} e\left(t \gamma_{\nu}-\beta_{\nu}\right) .
$$

For $P \in \mathbb{N}, b \in \mathbb{Z}, B \in \mathbb{R}, B>0$ let

$$
J:=\int_{b}^{b+B}|f(t)|^{2 P} d t .
$$


The multinomial theorem gives

$$
f(t)^{P}=\sum_{\substack{j_{-1}+\ldots+j_{k}=P \\ j_{\nu} \geq 0(\nu=-1,0, \ldots, k)}} \ldots \frac{P !}{j_{-1} ! \ldots j_{k} !} e\left(\sum_{\nu=-1}^{k} j_{\nu}\left(t \gamma_{\nu}-\beta_{\nu}\right)\right) .
$$

For $\alpha \in \mathbb{C}$ denote by $\bar{\alpha}$ the complex conjugate of $\alpha$; we have $|\alpha|^{2}=\alpha \bar{\alpha}$. For $x \in \mathbb{R}$ we have $\overline{e(x)}=e(-x)$. With

$$
\begin{gathered}
\mathbf{j}=\left(j_{-1}, \ldots, j_{k}\right) \in \mathbb{Z}^{k+2}, \quad \mathbf{j}^{\prime}=\left(j_{-1}^{\prime}, \ldots, j_{k}^{\prime}\right) \in \mathbb{Z}^{k+2}, \\
S\left(\mathbf{j}, \mathbf{j}^{\prime}\right):=\sum_{\nu=-1}^{k}\left(j_{\nu}-j_{\nu}^{\prime}\right) \gamma_{\nu}, \quad T\left(\mathbf{j}, \mathbf{j}^{\prime}\right):=\sum_{\nu=-1}^{k}\left(j_{\nu}-j_{\nu}^{\prime}\right) \beta_{\nu}
\end{gathered}
$$

we get

$$
J=\sum_{\substack{j_{-1}+\ldots+j_{k}=P \\ j_{\nu} \geq 0(\nu=-1, \ldots, k) \\ j_{-1}^{\prime}+\ldots+j_{k}^{\prime}=P \\ j_{\nu}^{\prime} \geq 0(\nu=-1, \ldots, k)}} \frac{P !}{j_{-1} ! \ldots j_{k} !} \frac{P !}{j_{-1}^{\prime} ! \ldots j_{k}^{\prime} !} \int_{b}^{b+B} e\left(S\left(\mathbf{j}, \mathbf{j}^{\prime}\right) t-T\left(\mathbf{j}, \mathbf{j}^{\prime}\right)\right) d t .
$$

We subdivide the multiple sum according as $\mathbf{j}=\mathbf{j}^{\prime}$ or $\mathbf{j} \neq \mathbf{j}^{\prime}$. We have $S(\mathbf{j}, \mathbf{j})=0, T(\mathbf{j}, \mathbf{j})=0$; but

$$
\begin{aligned}
S\left(\mathbf{j}, \mathbf{j}^{\prime}\right)=0 \Rightarrow & \exp \left(S\left(\mathbf{j}, \mathbf{j}^{\prime}\right)\right)=1 \\
\Rightarrow & \exp \left(\left(j_{0}-j_{0}^{\prime}\right) v\right) p_{1}^{\left(j_{1}-j_{1}^{\prime}\right) u_{1}} \ldots p_{k}^{\left(j_{k}-j_{k}^{\prime}\right) u_{k}}=1 \\
\Rightarrow & j_{\nu}=j_{\nu}^{\prime} \quad(\nu=0, \ldots, k) \\
& \left(\text { by } v u_{1} \ldots u_{k} \neq 0 \text { and by Lindemann }\right) \\
\Rightarrow & \mathbf{j}=\mathbf{j}^{\prime} \quad\left(\text { by } \quad \sum_{\nu=-1}^{k} j_{\nu}=\sum_{\nu=-1}^{k} j_{\nu}^{\prime}=P\right) ;
\end{aligned}
$$

we found

This gives

$$
\mathbf{j}=\mathbf{j}^{\prime} \Leftrightarrow S\left(\mathbf{j}, \mathbf{j}^{\prime}\right)=0
$$

$$
\begin{gathered}
\int_{b}^{b+B} e\left(S\left(\mathbf{j}, \mathbf{j}^{\prime}\right) t-T\left(\mathbf{j}, \mathbf{j}^{\prime}\right)\right) d t=B \quad\left(\mathbf{j}=\mathbf{j}^{\prime}\right), \\
\left|\int_{b}^{b+B} e\left(S\left(\mathbf{j}, \mathbf{j}^{\prime}\right) t-T\left(\mathbf{j}, \mathbf{j}^{\prime}\right)\right) d t\right| \leq \frac{1}{\pi\left|S\left(\mathbf{j}, \mathbf{j}^{\prime}\right)\right|} \quad\left(\mathbf{j} \neq \mathbf{j}^{\prime}\right) .
\end{gathered}
$$

For $\mathbf{j} \neq \mathbf{j}^{\prime}$ there exists by A. Baker (see [1, p. 22]) an effectively computable number $C\left(k, p_{k}\right)>0$ with

$$
\left|S\left(\mathbf{j}, \mathbf{j}^{\prime}\right)\right|^{-1}<A:=(2 P U v)^{C\left(k, p_{k}\right)} .
$$


We obtain

$$
\begin{aligned}
J \geq B \sum_{\substack{j_{-1}+\ldots+j_{k}=P \\
j_{\nu} \geq 0(\nu=-1, \ldots, k)}} \ldots \sum^{2} & \left(\frac{P !}{j_{-1} ! \ldots j_{k} !}\right)^{2} \\
& -\frac{A}{\pi} \sum_{\substack{j_{-1}+\ldots+j_{k}=P \\
j_{\nu} \geq 0(\nu=-1, \ldots, k) \\
j_{-1}^{\prime}+\ldots+j_{k}^{\prime}=P \\
j_{\nu} \geq 0(\nu=-1, \ldots, k)}} \frac{P !}{j_{-1} ! \ldots j_{k} !} \frac{P !}{j_{-1}^{\prime} ! \ldots j_{k}^{\prime} !}
\end{aligned}
$$

where in the second multiple sum we have dropped the condition $\mathbf{j} \neq \mathbf{j}^{\prime}$; to the first multiple sum we apply the Cauchy inequality and observe

$$
\sum_{\substack{j-1+\ldots+j_{k}=P \\ j_{\nu} \geq 0 \\(\nu=-1, \ldots, k)}} \ldots \sum^{k+2} .
$$

This gives

$$
J \geq\left(\frac{B}{(P+1)^{k+2}}-\frac{A}{\pi}\right)\left(\sum_{\substack{j_{-1}+\ldots+j_{k}=P \\ j_{\nu} \geq 0(\nu=-1, \ldots, k)}} \ldots \frac{P !}{j_{-1} ! \ldots j_{k} !}\right)^{2}
$$

since the last multiple sum equals $(1+\ldots+1)^{P}=(k+2)^{P}$, we have

$$
J \geq\left(\frac{B}{(P+1)^{k+2}}-\frac{A}{\pi}\right)(k+2)^{2 P} .
$$

For some $\tau \in \mathbb{R}, b \leq \tau \leq b+B$, we have

$$
|f(\tau)|=\sup _{b \leq t \leq b+B}|f(t)|
$$

this gives

$$
J \leq B|f(\tau)|^{2 P}
$$

We choose

$$
B:=A(P+1)^{k+2} .
$$

This gives

$$
\begin{gathered}
\frac{B}{2(P+1)^{k+2}}(k+2)^{2 P} \leq J \leq B|f(\tau)|^{2 P} \\
|f(\tau)| \geq(k+2) \exp \left(-\frac{k+2}{2 P} \log (2(P+1))\right) \\
>(k+2)\left(1-\frac{k+2}{2 P} \log (2(P+1))\right)
\end{gathered}
$$


But $\log (2(P+1)) \leq \frac{4}{3} \log P(P \in \mathbb{R}, P \geq 11)$. Setting

$$
\mu:=\frac{(k+2)^{2} \log P}{3 P}<1
$$

we obtain

$$
|f(\tau)|>k+2-2 \mu
$$

(1) implies

$$
f(t)=1+e\left(t \gamma_{\nu}-\beta_{\nu}\right)+\sum_{\substack{\mu=0 \\ \mu \neq \nu}}^{k} e\left(t \gamma_{\mu}-\beta_{\mu}\right)
$$

the triangle inequality and $|e(x)|=1(x \in \mathbb{R})$ give

$$
|f(t)| \leq k+\left|1+e\left(t \gamma_{\nu}-\beta_{\nu}\right)\right| \quad(\nu=0, \ldots, k, t \in \mathbb{R}) .
$$

We obtain

$$
\left|1+e\left(\tau \gamma_{\nu}-\beta_{\nu}\right)\right|>2-2 \mu
$$

and consequently

$$
\left|\sin \pi\left(\tau \gamma_{\nu}-\beta_{\nu}\right)\right|<\sqrt{2 \mu-\mu^{2}}<\sqrt{2 \mu} ;
$$

denote by $h_{\nu}$ the nearest integer to $\tau \gamma_{\nu}-\beta_{\nu}$; we have

$$
\left|\tau \gamma_{\nu}-\beta_{\nu}-h_{\nu}\right| \leq 1 / 2 \quad(\nu=0, \ldots, k) .
$$

Using

$$
\begin{gathered}
|\sin \pi x| \geq 2|x| \quad(x \in \mathbb{R},|x| \leq 1 / 2), \\
|\sin \pi(x+h)|=|\sin \pi x| \quad(x \in \mathbb{R}, h \in \mathbb{Z}),
\end{gathered}
$$

we obtain

$$
2\left|\tau \gamma_{\nu}-\beta_{\nu}-h_{\nu}\right| \leq\left|\sin \pi\left(\tau \gamma_{\nu}-\beta_{\nu}-h_{\nu}\right)\right|=\left|\sin \pi\left(\tau \gamma_{\nu}-\beta_{\nu}\right)\right|<\sqrt{2 \mu}
$$

$(\nu=0,1, \ldots, k)$; for $\nu=0$ this implies

$$
\left|\tau-h_{0}\right|<\sqrt{\mu} ;
$$

we replace $\tau$ by $h_{0}$ and with

$$
\gamma^{*}:=\sup _{\nu=1, \ldots, k}\left|\gamma_{\nu}\right|
$$

we get by the triangle inequality

$$
\left|h_{0} \gamma_{\nu}-\beta_{\nu}-h_{\nu}\right|<\mu^{*}:=\left(1+\gamma^{*}\right) \sqrt{\mu} \quad(\nu=1, \ldots, k) .
$$

Let $w \in \mathbb{R}, w \geq 1$; we are interested in the inequality

$$
\left|h_{0} \gamma_{\nu}-\beta_{\nu}-h_{\nu}\right|<1 / w
$$


with an effective estimate for $h_{0}$. We have

$$
\begin{gathered}
\gamma^{*} \leq U \log p_{k}, \quad \mu^{*}<3 U \sqrt{\mu} \log p_{k}, \\
\mu<\frac{(k+2)^{2}}{\sqrt{P}}, \quad \mu^{*}<\widetilde{\mu}:=\frac{3 U(k+2)}{\sqrt[4]{P}} \log p_{k} .
\end{gathered}
$$

The choice

$$
P:=\left[\left(3 w U(k+2) \log p_{k}\right)^{4}\right]+1
$$

implies $P \geq 11, \mu<1, \mu^{*} \leq 1 / w$. By $b \leq \tau \leq b+B, b \in \mathbb{Z}, h_{0} \in \mathbb{Z}$, $\left|\tau-h_{0}\right|<1$ we have $b \leq h_{0}<b+B+1$. By substitution, a bound for $B+1$ of the form $(2 U v w)^{C}$ can immediately be found. This proves

Theorem 1. Let $k \in \mathbb{N}, v \in \mathbb{N}, U \in \mathbb{R}, U \geq 1, b \in \mathbb{Z}, w \in \mathbb{R}, w \geq 1$. Let $p_{1}<\ldots<p_{k}$ be primes and

$$
u_{\nu} \in \mathbb{Z}, \quad 0<\left|u_{\nu}\right| \leq U, \quad \beta_{\nu} \in \mathbb{R} \quad(\nu=1, \ldots, k) .
$$

Then there exist $h_{\nu} \in \mathbb{Z}(\nu=0, \ldots, k)$ and an effectively computable number $C^{\prime}\left(k, p_{k}\right)>0$, depending on $k$ and $p_{k}$ only, with

$$
\left|h_{0} \frac{u_{\nu}}{v} \log p_{\nu}-\beta_{\nu}-h_{\nu}\right|<\frac{1}{w} \quad(\nu=1, \ldots, k)
$$

and

$$
b \leq h_{0} \leq b+(2 U v w)^{C^{\prime}\left(k, p_{k}\right)} .
$$

Theorem 1 is an effective Kronecker approximation theorem. If $p_{1}<$ $\ldots<p_{k}$ are the first $k$ primes, then $C^{\prime}\left(k, p_{k}\right)$ is an effectively computable $C^{\prime \prime}(k)$, depending on $k$ only.

Let $m \in \mathbb{N}$ and $r_{\nu} \in \mathbb{Z}, 0 \leq r_{\nu}<m(\nu=0, \ldots, k)$. (2) is equivalent to

$$
\left|\left(h_{0} m+r_{0}\right) \frac{u_{\nu}}{v} \log p_{\nu}-\left(\beta_{\nu} m+r_{0} \frac{u_{\nu}}{v} \log p_{\nu}-r_{\nu}\right)-\left(h_{\nu} m+r_{\nu}\right)\right|<\frac{m}{w} ;
$$

we write this as

$$
\left|h_{0}^{\prime} \frac{u_{\nu}}{v} \log p_{\nu}-\beta_{\nu}^{\prime}-h_{\nu}^{\prime}\right|<\frac{1}{w^{\prime}} \quad(\nu=1, \ldots, k) .
$$

Theorem 1 implies

Corollary 1. Let $k \in \mathbb{N}, v \in \mathbb{N}, U \in \mathbb{R}, U \geq 1, b \in \mathbb{Z}, w^{\prime} \in \mathbb{R}, w^{\prime} \geq 1$; let $p_{1}<\ldots<p_{k}$ be primes,

$$
u_{\nu} \in \mathbb{Z}, \quad 0<\left|u_{\nu}\right| \leq U, \quad \beta_{\nu}^{\prime} \in \mathbb{R} \quad(\nu=1, \ldots, k) ;
$$

furthermore, let $m \in \mathbb{N}$ and $r_{\nu} \in \mathbb{Z}, 0 \leq r_{\nu}<m \quad(\nu=0, \ldots, k)$. Then (3) holds with $h_{\nu}^{\prime} \equiv r_{\nu} \bmod m(\nu=0, \ldots, k)$ and

$$
b \leq h_{0}^{\prime} / m \leq 1+b+\left(2 U v m w^{\prime}\right)^{C^{\prime}\left(k, p_{k}\right)} .
$$


2. On a theorem of Motzkin. Let $n \in \mathbb{Z}, n>1$,

$$
\begin{gathered}
f(z):=\prod_{j=1}^{n-1}\left(z-z_{j}\right)=z^{n-1}+a_{1} z^{n-2}+\ldots+a_{n-1} \in \mathbb{C}[z], \\
d(f):=\inf _{j \neq k}\left\{1,\left|z_{j}-z_{k}\right|\right\}>0, \\
D(f):=\sup \left|z_{j}\right|, \quad K(f):=\sup \left|a_{j}\right| .
\end{gathered}
$$

We have $d(f) \leq 2 D(f)$ and

$$
\begin{gathered}
\left|a_{j}\right| \leq\left(\begin{array}{c}
n-1 \\
j
\end{array}\right)(1+D(f))^{j} \leq(2+D(f))^{n-1} \quad(j=1, \ldots, n-1), \\
K(f) \leq(2+D(f))^{n-1} .
\end{gathered}
$$

Lemma 1. Let $g \in \mathbb{N}$,

$$
\begin{gathered}
F(z)=\prod_{j=1}^{g}\left(z-\alpha_{j}\right) \in \mathbb{C}[z], \quad d(F)>0, \\
F^{*}(z) \in \mathbb{C}[z] \quad \text { with leading term } z^{g}, \\
\varrho \in \mathbb{R}, \quad 0<\varrho \leq \frac{1}{4} d(F)(<1) .
\end{gathered}
$$

For $j \in\{1, \ldots, g\}$ and

$$
B_{j}(F):=\left(\frac{d(F)}{2\left(\left|\alpha_{j}\right|+2\right)}\right)^{g-1}, \quad K\left(F^{*}-F\right) \leq \varrho B_{j}(F),
$$

there exist $\alpha_{j}^{*} \in \mathbb{C}$ with

$$
F^{*}\left(\alpha_{j}^{*}\right)=0 \quad \text { and } \quad\left|\alpha_{j}^{*}-\alpha_{j}\right|<\varrho ;
$$

furthermore,

$$
F^{*}(z)=\prod_{j=1}^{g}\left(z-\alpha_{j}^{*}\right), \quad d\left(F^{*}\right)>d(F) / 2 .
$$

In short: a small change in the coefficients of a polynomial implies a small change in its (simple) roots.

Proof. Let $j \in\{1, \ldots, g\}, \widetilde{z} \in \mathbb{C},\left|\widetilde{z}-\alpha_{j}\right|=\varrho$; then $|\widetilde{z}|<\left|\alpha_{j}\right|+1$ and $\left|\widetilde{z}-\alpha_{k}\right| \geq d(F)-\varrho>d(F) / 2(k \neq j)$. We get

$$
\begin{gathered}
\left|\left(F^{*}-F\right)(\widetilde{z})\right| \leq K\left(F^{*}-F\right)\left(1+|\widetilde{z}|+\ldots+|\widetilde{z}|^{g-1}\right) \\
<K\left(F^{*}-F\right)\left(\left|\alpha_{j}\right|+2\right)^{g-1}, \\
|F(\widetilde{z})|=\left|\widetilde{z}-\alpha_{j}\right| \prod_{k \neq j}\left|\widetilde{z}-\alpha_{k}\right| \geq \varrho\left(\frac{d(F)}{2}\right)^{g-1},
\end{gathered}
$$


and therefore

$$
\left|\left(F^{*}-F\right)(\widetilde{z})\right|<|F(\widetilde{z})|
$$

By Rouché's theorem, there exists exactly one $\alpha_{j}^{*} \in \mathbb{C}$ with $\left|\alpha_{j}^{*}-\alpha_{j}\right|<\varrho$ and $F^{*}\left(\alpha_{j}^{*}\right)=0$.

Let

$$
B(f):=\left(\frac{d(f)}{2(D(f)+2)}\right)^{n-1}=\inf _{j=1, \ldots, n-1} B_{j}(f) .
$$

Define $c_{j} \in \mathbb{R}$ by $a_{j}=c_{2 j-1}+i c_{2 j}(j=1, \ldots, n-1)$. Denote by $p_{j}$ the $j$ th prime; we have $p_{j}<(2 j)^{2} \quad(j=1,2, \ldots)$. Let

$$
\varrho \in \mathbb{R}, \quad 0<\varrho \leq d(f) / 4, \quad \varepsilon:=B(f) \varrho, \quad v:=\left[\frac{4 \log (4 n)^{2}}{\varepsilon}\right]+1 .
$$

Then

and there exist $u_{j} \in \mathbb{Z}$ such that for

$$
\frac{2}{v} \log p_{j}<\frac{\varepsilon}{2}
$$

$$
c_{j}^{*}:=\frac{2 u_{j}+1}{v} \log p_{j} \neq 0
$$

we have

$$
\left|c_{j}^{*}-c_{j}\right|<\varepsilon / 2 \quad(j=1, \ldots, 2 n-2) .
$$

Let

$$
\begin{gathered}
a_{j}^{*}:=c_{2 j-1}^{*}+i c_{2 j}^{*} \quad(j=1, \ldots, n-1), \\
f^{*}(z):=z^{n-1}+a_{1}^{*} z^{n-2}+\ldots+a_{n-1}^{*} .
\end{gathered}
$$

Then $K\left(f^{*}-f\right)<\varepsilon$. By Lemma 1 , there exist $z_{j}^{*} \in \mathbb{C}$ with

$$
f^{*}(z)=\prod_{j=1}^{n-1}\left(z-z_{j}^{*}\right), \quad\left|z_{j}^{*}-z_{j}\right|<\varrho \quad(j=1, \ldots, n-1),
$$

hence

$$
\begin{gathered}
\left|z_{j}^{*}-z_{k}^{*}\right|>d(f)-2 \varrho \quad(0<j<k<n), \\
d(f) / 2 \leq d(f)-2 \varrho<d\left(f^{*}\right)<d(f)+2 \varrho \leq 3 d(f) / 2 .
\end{gathered}
$$

Let $h_{0} \in \mathbb{Z}, z_{n}^{*}:=a_{1}^{*}-h_{0}, c_{2 n-1}^{*}:=0, c_{2 n}^{*}:=0, a_{n}^{*}:=c_{2 n-1}^{*}+i c_{2 n}^{*}$,

$$
g(z):=f^{*}(z)\left(z-z_{n}^{*}\right) \text {; }
$$

with

we have

$$
b_{j}:=a_{j}^{*}+a_{j-1}^{*}\left(h_{0}-a_{1}^{*}\right) \quad(j=2, \ldots, n)
$$

$$
g(z)-z^{n}-h_{0} z^{n-1}=b_{2} z^{n-2}+\ldots+b_{n} ;
$$


with

we have

$$
\begin{aligned}
\beta_{2 j-3} & :=-c_{2 j-1}^{*}+c_{2 j-3}^{*} c_{1}^{*}-c_{2 j-2}^{*} c_{2}^{*}, \\
\beta_{2 j-2} & :=-c_{2 j}^{*}+c_{2 j-2}^{*} c_{1}^{*}+c_{2 j-3}^{*} c_{2}^{*}
\end{aligned}
$$

$$
b_{j}=\left(h_{0} c_{2 j-3}^{*}-\beta_{2 j-3}\right)+i\left(h_{0} c_{2 j-2}^{*}-\beta_{2 j-2}\right) \quad(j=2, \ldots, n) .
$$

Let $w \in \mathbb{R}, w \geq 1$; we apply Theorem 1 with $k=2 n-2$ and obtain $h_{j} \in \mathbb{Z}$ $(j=0, \ldots, 2 n-2)$ such that for

$g^{*}(z):=z^{n}+h_{0} z^{n-1}+\left(h_{1}+i h_{2}\right) z^{n-2}+\ldots+\left(h_{2 n-3}+i h_{2 n-2}\right) \in(\mathbb{Z}[i])[z]$ we have

$$
K\left(g^{*}-g\right)<2 / w .
$$

By Corollary 1 with $m=9$ we can guarantee

$$
h_{0} \equiv h_{1} \equiv \ldots \equiv h_{2 n-3} \equiv 0 \bmod 9, \quad h_{2 n-2} \equiv 3 \bmod 9 .
$$

By the Schönemann-Eisenstein criterion for $3 \in \mathbb{Z}[i], g^{*}$ is irreducible over $\mathbb{Z}[i]$. Now

$$
\begin{aligned}
h_{0} \geq b:=[2 n(D(f)+1)]>0 & \Rightarrow h_{0}>2 \sum_{j=1}^{n-1}\left(\left|z_{j}\right|+1\right)+1 \geq 2 \sum_{j=1}^{n-1}\left|z_{j}^{*}\right|+1 \\
& \geq\left|\sum_{j=1}^{n-1} z_{j}^{*}\right|+\left|z_{k}^{*}\right|+1=\left|a_{1}^{*}\right|+\left|z_{k}^{*}\right|+1 \\
& \Rightarrow\left|z_{n}^{*}-z_{k}\right|>1 \quad(k=1, \ldots, n-1)
\end{aligned}
$$

hence

$$
d(g)=d\left(f^{*}\right) .
$$

Let $\sigma \in \mathbb{R}, 0<\sigma \leq d(g) / 4$; we have

$$
\begin{aligned}
B_{j}(g) & =\left(\frac{d(g)}{2\left(\left|z_{j}^{*}\right|+2\right)}\right)^{n-1} \quad(j=1, \ldots, n-1) \\
& >\widetilde{B}(f):=\left(\frac{d(f)}{4(D(f)+3)}\right)^{n-1} ;
\end{aligned}
$$

let

$$
w:=\frac{2}{\sigma \widetilde{B}(f)} .
$$

By Lemma 1, there exists $\zeta_{j} \in \mathbb{C}$ with

$$
g^{*}\left(\zeta_{j}\right)=0, \quad\left|\zeta_{j}-z_{j}^{*}\right|<\sigma \quad(j=1, \ldots, n-1),
$$

hence

$$
\left|\zeta_{j}-\zeta_{k}\right|>d\left(f^{*}\right)-2 \sigma \quad(0<j<k<n) .
$$


Let $\eta \in \mathbb{R}, 0<\eta \leq d(f) / 4, \varrho:=\eta / 2$; then

$$
\begin{gathered}
\left|\zeta_{j}-z_{j}\right|<\eta \quad(j=1, \ldots, n-1), \\
\left|\zeta_{j}-\zeta_{k}\right|>d(f)-2 \varrho-2 \sigma>d(f) / 2 \quad(0<j<k<n)
\end{gathered}
$$

and obviously $\varrho \leq d(f) / 8<d(f) / 4$,

$$
\sigma:=\frac{\eta}{2} \leq \frac{d(f)}{8}<\frac{d\left(f^{*}\right)}{4}=\frac{d(g)}{4} .
$$

In $c_{j}^{*}$ we certainly have

$$
0<\left|2 u_{j}+1\right| \leq 2 v(K(f)+1) \leq U:=2 v(3+D(f))^{n-1} .
$$

In Corollary 1 we have

$$
0<b \leq h_{0} / 9 \leq b+(2 U v w)^{5 C^{\prime \prime}(2 n-2)} ;
$$

substitution gives

$$
\left|h_{0}\right|<2\left(2 \cdot 2 v^{2}(3+D(f))^{n-1} w\right)^{5 C^{\prime \prime}}
$$

but

$$
0<v<\frac{\log (4 n)^{2}}{B(f) \eta} \cdot 16
$$

so the estimate for $\left|h_{0}\right|$ takes the form

$$
\left|h_{0}\right|<\left(L(n, d(f), D(f)) \eta^{-3}\right)^{5 C^{\prime \prime}}
$$

where $L>0$ is increasing in $n, 1 / d(f)$ and $D(f)$. For

$$
S:=\sup \left|a_{j}\right|, \quad S^{\prime}:=\sup \left|a_{j}^{*}\right|, \quad S^{\prime \prime}:=\sup \left|b_{j}\right|
$$

we have

$$
\begin{gathered}
S^{\prime}<S+1 \quad\left(\text { since } K\left(f^{*}-f\right)<1\right), \\
S^{\prime \prime}<S^{\prime}+S^{\prime}\left(\left|h_{0}\right|+S^{\prime}\right) \quad\left(\text { by definition of } b_{j}\right), \\
\left|h_{2 j-1}+h_{2 j} i\right|<S^{\prime \prime}+1 \quad(j=1, \ldots, n-1)\left(\text { since } K\left(g^{*}-g\right)<1\right)
\end{gathered}
$$

and $g^{*}$ is effectively computable. This completes the proof of

Theorem 2. Let $n \in \mathbb{Z}, n>1, z_{j} \in \mathbb{C}(j=1, \ldots, n-1)$,

$$
d:=\inf _{j \neq k}\left\{1,\left|z_{j}-z_{k}\right|\right\}>0, \quad D:=\sup \left|z_{j}\right|, \eta \in \mathbb{R}, 0<\eta \leq d / 4 .
$$

Then there exists an effectively computable polynomial $g^{*}(z)=z^{n}+$ $e_{1} z^{n-1}+\ldots+e_{n}$ with $e_{j} \in \mathbb{Z}[i]$ and with the properties:

(i) $g^{*}$ is irreducible over $\mathbb{Z}[i]$,

(ii) its suitably numbered roots $\zeta_{1}, \ldots, \zeta_{n}$ satisfy

$$
\left|\zeta_{j}-z_{j}\right|<\eta \quad(j=1, \ldots, n-1) .
$$

This is a refinement of a result of Motzkin [2] who has no upper bound for $\left|\zeta_{n}\right|$. 
TheOREM 3. If in Theorem 2 the set $\left\{z_{1}, \ldots, z_{n-1}\right\}$ is symmetric about $\mathbb{R} \subset \mathbb{C}$, we have $e_{j} \in \mathbb{Z} \quad(j=1, \ldots, n)$ (and $\zeta_{1}, \ldots, \zeta_{n}$ is a complete set of conjugate algebraic integers).

Proof. In the proof of Theorem 2 we have

$$
f(z) \in \mathbb{R}[z], \quad a_{j}^{*}=c_{2 j-1}^{*} \quad(j=1, \ldots, n-1),
$$

$f^{*}(z) \in \mathbb{R}[z], \quad\left\{z_{1}^{*}, \ldots, z_{n-1}^{*}\right\}$ symmetric about $\mathbb{R}$,

$$
z_{n}^{*}:=a_{1}^{*}-h_{0} \in \mathbb{R}, \quad g(z) \in \mathbb{R}[z], \quad g^{*}(z) \in \mathbb{Z}[z] .
$$

\section{References}

[1] A. Baker, Transcendental Number Theory, Cambridge Univ. Press, 1975.

[2] T. Motzkin, From among $n$ conjugate algebraic integers, $n-1$ can be approximately given, Bull. Amer. Math. Soc. 53 (1947), 156-162.

[3] W. Narkiewicz, Elementary and Analytic Theory of Algebraic Numbers, PWN, Warszawa 1974.

[4] P. Turán, Nachtrag zu meiner Abhandlung "On some approximative Dirichlet polynomials in the theory of zeta-function of Riemann", Acta Math. Acad. Sci. Hungar. 10 (1959), 277-298.

INSTITUT FÜR MATHEMATIK

UNIVERSITÄT HANNOVER

D-3000 HANNOVER, GERMANY

Received on 21.2.1992

and in revised form on 8.5.1992 